Mathematical Modelling and Analysis

Volume 22 Number 1, January 2017, 21-36

https://doi.org/10.3846/13926292.2017.1258678

(c) Vilnius Gediminas Technical University, 2017
Publisher: Taylor\&Francis and VGTU

http://www.tandfonline.com/TMMA

ISSN: $1392-6292$

eISSN: 1648-3510

\title{
Asymptotic Behavior for Radially Symmetric Solutions of a Logistic Equation with a Free Boundary
}

\section{Jingjing Cai and Quanjun Wu}

School of Mathematics and Physics, Shanghai University of Electric Power

Pingliang Road 2103, 200090 Shanghai, China

E-mail(corresp.): cjjing1983@163.com

E-mail: wuquanjun2008@163.com

Received March 4, 2016; revised November 1, 2016; published online January 5, 2017

\begin{abstract}
In this paper we investigate a logistic equation with a new free boundary condition appearing in ecology, we aim to describe the spreading of a new or invasive species by studying the asymptotic behavior of the radially symmetric solutions of the problem. We will obtain a trichotomy result: spreading (the solution converges to a stationary solution defined on the half-line), transition (the solution converges to a stationary solution with compact support) and vanishing (the solution converges to 0 within a finite time). Besides we can also obtain a dichotomy result (either spreading or vanishing happens). Moreover, in the spreading case, we give the sharp estimate of the asymptotic spreading speed of the free boundary.
\end{abstract}

Keywords: asymptotic behavior of solutions, logistic equation, free boundary, trichotomy result.

AMS Subject Classification: 35K20; 35K55; 35B40; 35R35.

\section{Introduction}

We are interested in the asymptotic behavior of the radially symmetric solution $u(t, r)\left(r=|x|, x=\left(x_{1}, x_{2}, \ldots, x_{N}\right) \in \mathbb{R}^{N}, N>1\right)$ of the following logistic equation with a free boundary:

$$
\begin{cases}u_{t}=\Delta u+u(1-u), & R<r<h(t), t>0, \\ u(t, R)=u(t, h(t))=0, & t>0, \\ h(t) h^{\prime}(t)=-u_{r}(t, h(t))-\alpha, & t>0 \\ h(0)=h_{0}, \quad u(0, r)=u_{0}(r), & R \leq r \leq h_{0},\end{cases}
$$

where $\Delta u=u_{r r}+\frac{N-1}{r} u_{r}, r=h(t)$ is a moving boundary to be determined together with $u(t,|x|), R>0, h_{0}>R$ and $\alpha \in \mathbb{R}$ are given constants. The initial function $u_{0}$ belongs to $\mathcal{J}\left(h_{0}\right)$, where

$$
\mathcal{J}\left(h_{0}\right):=\left\{\phi \in C^{2}\left(\left[R, h_{0}\right]\right): \phi(R)=\phi\left(h_{0}\right)=0, \phi(r) \geq(\not \equiv) 0 \text { in }\left(R, h_{0}\right)\right\} .
$$


Problem (1.1) can be used to model the spreading of a new or invasive species. Here $u(t,|x|)$ denotes the population density over an $N$-dimensional habitat, which is radically symmetric. The initial function $u_{0}(|x|)$ stands for the population in its early stage of introduction. Its spreading front is represented by the free boundary $|x|=h(t)$, which is a sphere $\partial B_{h(t)}$ with radius $h(t)$ growing at a speed $\frac{1}{2}\left(h^{2}(t)\right)^{\prime}=-u_{r}(t, h(t))-\alpha$. We mainly study the problem (1.1) with $\alpha \in \mathbb{R}$, with $\alpha$ representing the decay rate of the species caused by the environment at the boundary, such as food, the climate, other species and so on. When $\alpha>0$, such a boundary condition means that there is a spreading resistant force at the front for some species. Intuitively, the presence of $\alpha>0$ makes the solution more difficult to spread than the case where $\alpha=0$. Indeed, $h^{\prime}(t)>0$ if and only if $u_{r}(t, h(t))<-\alpha$. When $\alpha<0$, the boundary condition means that the environment at the free boundary is favorable for spreading of the species. Indeed, the boundary condition we used can be derived by the competition-diffusion system (see $[14,15]$ for details).

Moreover, such a boundary condition is also widely used in many biological models. For example, $[4,12,18]$ studied a protocell model. It mimics the biological process of growth and dissolution of an organism under external nutrient supply. Besides, Friedman [11] used such a free boundary condition to describe the condensation and evaporation of a isolated drop in three dimensional space. $r=h(t)$ represents the radius of the drop. When condensation happens, $\alpha<0$, then the isolated drop becomes larger; in the case of evaporation, $\alpha>0$, and the the isolated drop becomes smaller.

Problem (1.1) with $\alpha=0$ and $h^{\prime}(t)=-u_{r}(t, h(t))$ was studied by [5,6,7, $8,10,16,17]$. When $N=1$, [17] used this model to describe the spreading of a new or invasive species, and obtained a dichotomy result: the species either spreads to the whole environment and stabilizes at the positive state defined on $[0,+\infty)$ or it vanishes $(u \rightarrow 0)$ as time goes to infinity. Later, they studied the corresponding problem of (1.1) with $\alpha=0$ in higher dimension spaces (see [16]). Moreover, [5,6] also obtained a spreading-vanishing dichotomy result by studying the problem (1.1) with $u(t, R)=0$ being replaced by $u_{r}(t, 0)=0$. Besides the asymptotic behavior of the solution, another interesting problem is the asymptotic spreading speed of the free boundary when spreading happens. $[7,8,10]$ proved that, when spreading happens for a solution $(u, h)$ of the problem (1.1) with $\alpha=0, N=1$ and when the free boundary condition is $h^{\prime}(t)=-u_{x}(t, h(t))$,

$$
c^{*}:=\lim _{t \rightarrow \infty} \frac{h(t)}{t}>0 .
$$

Recently, [5] obtained (1.2) for $N \geq 2$, and [2] also proved that (1.2) holds when the free boundary satisfies the condition $h^{\prime}(t)=-u_{x}(t, h(t))-\alpha$ with a small $\alpha>0$.

In this paper, we mainly study the asymptotic behavior of solutions and the asymptotic spreading speed of free boundary when spreading happens. Using the similar arguments as in $[2,7]$, we have the following basic results:

(a) For any given $\gamma \in(0,1)$, there is a $T \in(0,+\infty)$ such that the free boundary problem $(1.1)$ has a solution $(u, h) \in C^{\frac{1+\gamma}{2}, 1+\gamma}\left(\bar{\Omega}_{T}\right) \times C^{1+\gamma / 2}([0, T])$, where $\Omega_{T}:=\left\{(t, r) \in \mathbb{R}^{2}: r \in[R, h(t)], t \in(0, T]\right\}$, and the solution can be 
extended to some interval $\left(0, T_{0}\right)$ with $T_{0}>T$ as long as $\inf _{0<t<T} h(t)>R$.

(b) The limit $\lim _{t \rightarrow T} h(t)$ exists, denote it by $h_{\infty}$.

We will see in the next section that the asymptotic behavior of the solutions is influenced by $\alpha$. (i) There is a critical value $\alpha_{0}:=\sqrt{2 \int_{0}^{1} s(1-s) d s}=\frac{1}{\sqrt{3}}$ such that $u$ converges to 0 and $h(t)$ shrinks to $R$ within a finite time when $\alpha \geq \alpha_{0}$; (ii) when $0<\alpha<\alpha_{0}$, we will obtain a trichotomy result for the solution $u(t, r)$ of (1.1) (see Theorem 1$)$ : spreading ( $u$ converges to a stationary solution defined on $[R, \infty)$ ), vanishing ( $u$ converges to 0 within a finite time) and transition ( $u$ converges to a stationary solution with compact support); (iii) in the case where $\alpha=0$, we will show that, for the solution $u(t, r)$, either vanishing or spreading happens; (iv) when $\alpha<0$, only spreading happens. We give the details in the following theorems.

Theorem 1. (The case $\left.0<\alpha<\alpha_{0}\right)$. Assume that $0<\alpha<\alpha_{0}$ and $(u, h)$ is a solution of (1.1) defined on the time maximal interval $(0, T)$. Then either

(i) spreading: $T=+\infty, h_{\infty}=+\infty$ and

$$
\lim _{t \rightarrow \infty} u(t, r)=V(r) \text { locally uniformly in }[R,+\infty),
$$

where $V$ is the unique positive solution of

$$
\left\{\begin{array}{l}
\Delta v+v(1-v)=0, r>R \\
v(R)=0
\end{array}\right.
$$

or

(ii) vanishing: $T<+\infty$, $\lim _{t \rightarrow T} h(t)=R$ and

$$
\lim _{t \rightarrow T} \max _{R \leq r \leq h(t)} u(t, r)=0
$$

or

(iii) transition: $T=+\infty, h_{\infty}=l_{\alpha}>R$ and

$$
\lim _{t \rightarrow \infty} u(t, \cdot)=v_{\alpha}(\cdot) \text { locally uniformly in }\left(R, h_{\infty}\right) \text {, }
$$

where $(l, v)=\left(l_{\alpha}, v_{\alpha}\right)$ is the unique positive solution of

$$
\left\{\begin{array}{l}
\Delta v+v(1-v)=0, \quad r \in(R, l), \\
v(R)=v(l)=0,-v^{\prime}(l)=\alpha .
\end{array}\right.
$$

Remark 1. There is no transition result as in (iii) when $\alpha \leq 0$ or $\alpha \geq \alpha_{0}$. Moreover, when $\alpha \rightarrow 0$, one can prove that $v_{\alpha} \rightarrow 0$ and $l_{\alpha} \rightarrow R^{*}$, where $R^{*}>R$ is determined by the following eigenvalue problem

$$
\left\{\begin{array}{l}
-\Delta \phi=\lambda \phi, \quad R<r<l \\
\phi(R)=\phi(l)=0 .
\end{array}\right.
$$

It is well known that (cf. [5]) there is a unique $R^{*}>0$ such that the principal eigenvalue $\lambda_{1}$ of the above problem satisfies $\lambda_{1}=1$ when $l=R^{*}$, and $1>\lambda_{1}$ when $l>R^{*}$. The conclusion in Theorem 1 (iii) gives an explanation of [16, Theorem 4], i.e., vanishing happens if $h_{\infty} \leq R^{*}$. 
Remark 2. We now give some explanations of the main results. Assume the spreading resistant force at the boundary is not large (i.e., $\alpha<\alpha_{0}$ ), combining this and the sufficient conditions in Section 3, we have the following three cases for the spreading of the species. (a) in the spreading case, the species can expands to the entire space when the initial density of the species is sufficiently large; (b) in the vanishing case, the species can not spread and it disappears in finite time when the initial density is small; (c) the last case means that the species converges to a stationary state with a certain radius.

Theorem 2. (The case $\left.\alpha \geq \alpha_{0}, \alpha \leq 0\right)$. Assume $(u, h)$ is a solution of (1.1) defined on the maximal interval $(0, T)$. Then (i) when $\alpha \geq \alpha_{0}$, only vanishing happens, (ii) when $\alpha<0$, only spreading happens, (iii) when $\alpha=0$, we have a dichotomy result: either spreading happens or

$$
T=+\infty, R<h_{\infty}<+\infty, \quad \lim _{t \rightarrow \infty} \max _{R<r<h(t)} u(r, t)=0 .
$$

Remark 3. From this theorem, we see that (1.9) is different from (1.5) since $h_{\infty} \neq R$ and $T=+\infty$ here. We next give an explanation of the theorem. Ecologically, when the spreading resistant force at the boundary is large (or, the environment at the boundary is bad), i.e., $\alpha>\alpha_{0}$, the species shrinks and disappears in finite time for any initial density. On the other hand, $\alpha<0$ means that the environment at the free boundary is favorable for spreading, so the species will always spreads.

Finally, we show that when spreading happens (see Theorem 1 (i), Theorem 2 (ii)-(iii)), the asymptotic spreading speed of the free boundary $h(t)$ has the following properties.

Theorem 3. Assume that spreading happens for the solution $(u, h)$ of (1.1), then

$$
\lim _{t \rightarrow \infty} \frac{h(t)}{\sqrt{t}}=\sqrt{2\left(\alpha_{0}-\alpha\right)}
$$

In this theorem, the limit in (1.10) gives the relation between the asymptotic spreading speed and $\alpha$. We can easily derive that the free boundary $h(t)$ satisfies $\lim _{t \rightarrow \infty} \frac{h(t)}{t}=0$. Moreover, (1.10) implies that the spreading speed of $h(t)$ is decreasing in $\alpha$. Therefore, the spreading front $|x|=h(t)$ spreads fast if the spreading resistant force at the boundary is small (i.e., $\alpha$ is small).

\section{Proof of Main Theorems}

In this section, we first give some basic results which play key roles in our approach, then prove Theorem 1 - Theorem 3 .

We first consider the radially symmetric solutions for the following elliptic problem which will be used later:

$$
\left\{\begin{array}{l}
\Delta v+v(1-v)=0, v(r)>0, R<r<l \\
v(R)=v(l)=0
\end{array}\right.
$$


It is well known that the solution $v$ of (2.1) has the following properties (cf. $[3,5])$ : (i) when $l \leq R^{*}, v \equiv 0$ is the unique nonnegative solution of $(2.1)$, (ii) when $l>R^{*},(2.1)$ has a unique positive solution $v(r)$, where the definition of $R^{*}$ is given by Remark 1 .

We now prepare the following comparison theorem which will be used to investigate the asymptotic behavior of solutions for the problem (1.1). The proof of this lemma is identical to that of [7, Lemma 5.7].

Lemma 1. Suppose that $T \in(0, \infty), \bar{h} \in C^{1}([0, T]), \bar{u} \in C\left(\bar{\Omega}_{T}\right) \cap C^{1,2}\left(\Omega_{T}\right)$ with $\Omega_{T}=\left\{(t, r) \in \mathbb{R}^{2}: 0<t \leq T, R<r<\bar{h}(t)\right\}$, and

$$
\begin{cases}\bar{u}_{t} \geq \Delta \bar{u}+\bar{u}(1-\bar{u}), & 0<t \leq T, \quad R<r<\bar{h}(t), \\ \bar{u}(t, R) \geq 0, \bar{u}(t, \bar{h}(t))=0, & 0<t \leq T, \\ \bar{h}(t) \bar{h}^{\prime}(t) \geq-\bar{u}_{r}(t, \bar{h}(t))-\alpha, & 0<t \leq T .\end{cases}
$$

If $h_{0} \leq \bar{h}(0), u_{0}(r) \leq \bar{u}(0, r)$ in $\left[R, h_{0}\right]$, and if $(u, h)$ is a solution of (1.1) with initial data $u_{0}(r)$. Then

$$
h(t) \leq \bar{h}(t), u(r, t) \leq \bar{u}(r, t) \text { for } t \in(0, T] \text { and } r \in(R, h(t)) .
$$

Remark 4. The pair $(\bar{u}, \bar{h})$ is usually called an upper solution of the problem (1.1) and one can define a lower solution by revising all the inequalities.

\subsection{Proof of Theorem 1}

(i) Assume that $(u, h)$ is a solution of (1.1). If $h_{\infty}=+\infty$, then we prove that (1.3) holds. Choose a bounded continuous function $W_{0}(r) \geq 0$ for $r \in[R,+\infty)$, $W_{0}(r) \geq u_{0}(r)$ for $r \in\left[R, h_{0}\right]$ and $W_{0}(R)=0$. Let $W(t, r)$ be the radically symmetric solution of

$$
\begin{cases}W_{t}=\Delta W+W(1-W), & t>0, r>R \\ W(t, R)=0, & t>0 \\ W(0, r)=W_{0}(r), & r>R\end{cases}
$$

It is well known that

$$
\lim _{t \rightarrow \infty} W(t, r)=V(r) \text { locally uniformly in }[R,+\infty),
$$

where $V(r)$ is the unique positive solution of (1.4). By the comparison principle, we can derive that

$$
u(t, r) \leq W(t, r) \quad \text { for }(t, r) \in(0,+\infty) \times(R, h(t)) .
$$

By (2.2) and (2.3) we have

$$
\limsup _{t \rightarrow \infty} u(t, r) \leq V(r) \text { for } r \in[R,+\infty) .
$$

On the other hand, since $h_{\infty}=+\infty$, for any large $l>R^{*}$, there is $\tau>0$ such that $h(\tau)=l$ and $h(t) \geq l$ for all $t>\tau$. Let $\underline{u}_{l}(t, r)$ be the solution of the following problem

$$
\begin{cases}\underline{u}_{t}=\Delta \underline{u}+\underline{u}(1-\underline{u}), & t>\tau, R<r<l, \\ \underline{u}(t, R)=\underline{u}(t, l)=0, & t>\tau, \\ \underline{u}(0, r)=\psi(r), & R<r<l\end{cases}
$$


where $\psi$ is a nonnegative continuous function satisfying $\psi(R)=\psi(l)=0$ and $\psi(r) \leq u(\tau, r)$ for $R<r<l$. Then the comparison principle implies

$$
\underline{u}_{l}(t, r) \leq u(t, r) \text { for } t>\tau, R \leq r \leq l .
$$

By [3] one can obtain

$$
\lim _{t \rightarrow \infty} \underline{u}_{l}(t, r)=v_{l}(r) \text { uniformly in }[0, l],
$$

where $v_{l}$ is the unique positive solution of

$$
\left\{\begin{array}{l}
\Delta v+v(1-v)=0, \quad R<r<l, \\
v(R)=v(l)=0 .
\end{array}\right.
$$

It is well known that $\lim _{l \rightarrow \infty} v_{l}(r)=V(r)$ (cf. [5]). Hence, combining this, (2.5) and (2.6), we have

$$
V(r) \leq \liminf _{t \rightarrow \infty} u(t, r) \text { for } r \in[R,+\infty) .
$$

Therefore, it follows from (2.4) and (2.7) that

$$
\lim _{t \rightarrow \infty} u(t, r)=V(r) \text { locally uniformly in }[R,+\infty) .
$$

(ii) Assume that $\lim _{t \rightarrow T} h(t)=R$, then we prove that $T<+\infty$ and (1.5) holds. By $[1,7]$ one can prove that there exists a constant $C_{1}$ independent of $t$ such that $u(t, r) \leq C_{1}$. In order to prove that $u$ converges to 0 , we need to construct the function

$$
U(t, r):=C_{1}\left[2 M(h(t)-r)-M^{2}(h(t)-r)^{2}\right]
$$

over the region $Q:=\left\{(t, r): 0<t<T, \max \left\{h(t)-M^{-1}, R\right\}<r<h(t)\right\}$, where

$$
M:=\max \left\{\frac{1}{2}\left(\alpha R+\sqrt{\alpha^{2} / R^{2}+2}\right), \frac{4}{3 C_{1}}\left\|u_{0}\right\|_{C^{1}\left(\left[R, h_{0}\right]\right)}\right\} .
$$

Clearly $0 \leq U \leq C_{1}$ in $Q$. We now show that $U(t, r)$ is an upper solution of (1.1) in $Q$. By the Hopf lemma, we have

$$
h(t) h^{\prime}(t)=-u_{r}(t, h(t))-\alpha>-\alpha,
$$

so $h^{\prime}(t)>-\alpha / R$. On the other hand, it follows from $r>h(t)-M^{-1}$ that $U_{r}=-2 C_{1} M-2 C_{1} M^{2}[r-h(t)]<0$. Therefore, by the definitions of $U$ and $M$ we have

$$
\begin{aligned}
U_{t}-U_{r r}-\frac{(N-1) U_{r}}{r}-U(1-U) & \geq U_{t}-U_{r r}-U(1-U) \\
& \geq C_{1}\left(2 M^{2}-2 M \alpha / R-1\right) \geq 0 \text { in } Q .
\end{aligned}
$$

Moreover,

$$
U(t, h(t))=u(t, h(t))=0 \quad \text { for } t \in(0, T)
$$


and

$$
U(t, R)>0=u(t, R), \quad \text { when } h(t)-M^{-1} \leq R .
$$

Therefore, $u(t, r) \leq U(t, r)$ in $Q$ by the comparison principle. Note that $\lim _{t \rightarrow T} h(t)=R$, then there exists $T_{1}<T$ such that $h(t)-M^{-1}<R$ for $t>T_{1}$. Therefore, $u(t, r) \leq U(t, r)$ for $t>T_{1}$ and $r \in[R, h(t)]$. For such $t$ and $r$ we have

$$
U(t, r) \leq 2 M C_{1}(h(t)-R) \rightarrow 0 \quad \text { as } \quad t \rightarrow T
$$

it follows that

$$
\|u(t, \cdot)\|_{L^{\infty}([R, h(t)])} \rightarrow 0 \quad \text { as } \quad t \rightarrow T .
$$

We now prove that $T<+\infty$. By $\lim _{t \rightarrow T} h(t)=R$, there is some $H>R$ such that $h(t) \leq H$ for $t \in[0, T)$. Set $L:=2(1+H)$ and

$$
\xi_{0}(r):=2 \varepsilon\left(1-r^{2} / L^{2}\right),
$$

where $\varepsilon>0$ is small such that

$$
16\left(\alpha / R+\sqrt{\alpha^{2} / R^{2}+2}\right) \varepsilon \leq \alpha, \quad 48 \varepsilon \leq \alpha .
$$

Consider the problem

$$
\begin{cases}\xi_{t}=\Delta \xi+2 \xi(1-\xi / 2 \varepsilon), & R<r<\bar{h}(t), t>0 \\ \xi(t, R)=\xi(t, \bar{h}(t))=0, & t>0 \\ \bar{h}(t) \bar{h}^{\prime}(t)=-\xi_{r}(t, \bar{h}(t))-\alpha, & t>0 \\ \bar{h}(0)=L, \quad \xi(0, r)=\xi_{0}(r), & R \leq r \leq L\end{cases}
$$

It is obvious that $2 \xi\left(1-\frac{\xi}{2 \varepsilon}\right)>\xi(1-\xi)$ for $\xi \in[0, \varepsilon]$. By the definition of $\xi_{0}(r)$, we have $\xi(t, r) \leq 2 \varepsilon$ for all $t \geq 0$. Construct a function

$$
U^{\varepsilon}(t, r):=4 \varepsilon\left[2 M(\bar{h}(t)-r)-M^{2}(\bar{h}(t)-r)^{2}\right]
$$

over $\bar{Q}:=\left\{(t, r): t>0, \max \left\{R, \bar{h}(t)-M^{-1}\right\} \leq r \leq \bar{h}(t)\right\}$, where $M:=$ $\max \left\{\alpha / R+\sqrt{\alpha^{2} / R^{2}+2}, 3\right\}$. Then $U^{\varepsilon}(t, r)$ is an upper solution of (2.9) over $\bar{Q}$ and so

$$
-\xi_{r}(t, \bar{h}(t)) \leq-U_{r}^{\varepsilon}(t, \bar{h}(t))=8 M \varepsilon \leq \alpha / 2 .
$$

Therefore, $\bar{h}(t) \bar{h}^{\prime}(t) \leq-\alpha / 2$. Thus $\bar{h}(t) \rightarrow R$ as $t \rightarrow \bar{T}^{*} \leq\left(L^{2}-R^{2}\right) / \alpha$.

On the other hand, (2.8) implies that there exists some $T_{*} \in(0, T)$ such that $u(t, r) \leq \varepsilon$ for all $r \in[R, h(t)]$ and $t \geq T_{*}$. Clearly $\xi_{0}(r) \geq \varepsilon \geq u\left(T_{*}, r\right)$ for $r \in\left[R, h\left(T_{*}\right)\right]$. By the comparison principle we have $h\left(t+T_{*}\right) \leq \bar{h}(t)$ for $t>0$, and so $T$ can not be $\infty$.

(iii) Assume $R<h_{\infty}<+\infty$, then we prove that $h_{\infty}=l_{\alpha}$ and (1.6) holds. By the definition of $h_{\infty}$, for any $\varepsilon>0$, there exists $t^{*}>0$ such that $h_{\infty}-\varepsilon<h(t)<h_{\infty}+\varepsilon$ for $t>t^{*}$. Let $\bar{u}_{0}(r)$ be a $C^{2}$ function defined on $\left(R, h_{\infty}+\varepsilon\right)$ and it satisfies

$$
\bar{u}_{0}(r) \geq u\left(t^{*}, r\right) \text { for } r \in\left(R, h\left(t^{*}\right)\right) \text { and } \bar{u}_{0}(R)=\bar{u}_{0}\left(h_{\infty}+\varepsilon\right)=0 .
$$

By the comparison principle we have

$$
u(t, r) \leq \bar{u}(t, r) \quad \text { for }(t, r) \in\left(t^{*}, \infty\right) \times(R, h(t)),
$$


where $\bar{u}(t, r)$ is the solution of

$$
\begin{cases}\bar{u}_{t}=\Delta \bar{u}+\bar{u}(1-\bar{u}), & t>t^{*}, R<r<h_{\infty}+\varepsilon, \\ \bar{u}(t, R)=\bar{u}\left(t, h_{\infty}+\varepsilon\right)=0, & t>t^{*} \\ \bar{u}\left(t^{*}, r\right)=\bar{u}_{0}(r), & R<r<h_{\infty}+\varepsilon .\end{cases}
$$

By [3, Corollary 3.4] we have

(a) when $h_{\infty}+\varepsilon \leq R^{*}, \lim _{t \rightarrow \infty} \bar{u}(t, \cdot)=0$ locally uniformly in $\left(R, h_{\infty}+\varepsilon\right)$;

(b) when $h_{\infty}+\varepsilon>R^{*}, \lim _{t \rightarrow \infty} \bar{u}(t, \cdot) \rightarrow \bar{u}_{\varepsilon}^{*}(\cdot)$ locally uniformly in $\left(R, h_{\infty}+\varepsilon\right)$,

where $\bar{u}_{\varepsilon}^{*}(r)$ is a positive function. More precisely, when $h_{\infty}+\varepsilon>R^{*}$, it follows from Corollary 3.4 of [3] that $\bar{u}_{\varepsilon}^{*}(r)$ is the unique positive solution of

$$
\left\{\begin{array}{l}
\Delta v+v(1-v)=0, \quad R<r<h_{\infty}+\varepsilon \\
v\left(h_{\infty}+\varepsilon\right)=v(R)=0 .
\end{array}\right.
$$

Hence, by (2.10) we have

$$
\lim _{t \rightarrow \infty} u(t, r)=0, \quad \text { or } \limsup _{t \rightarrow \infty} u(t, r) \leq \bar{u}_{\varepsilon}^{*}(r) \text { for } R<r<h_{\infty} .
$$

Similarly

$$
\liminf _{t \rightarrow \infty} u(t, r) \geq \underline{u}_{\varepsilon}^{*}(r) \text { for } R<r<h_{\infty}-\varepsilon, \text { when } h_{\infty}-\varepsilon>R^{*},
$$

where $\underline{u}_{\varepsilon}^{*}(r)$ is the unique positive solution of

$$
\left\{\begin{array}{l}
\Delta v+v(1-v)=0, \quad R<r<h_{\infty}-\varepsilon \\
v\left(h_{\infty}-\varepsilon\right)=v(R)=0 .
\end{array}\right.
$$

Moreover, we consider the case that $h_{\infty}=R^{*}$. By the properties of the solution of (2.1), a standard compactness and uniqueness argument, one can see that $\bar{u}_{\varepsilon}^{*} \rightarrow 0$ as $\varepsilon \rightarrow 0^{+}$when $h_{\infty}=R^{*}$. Hence, letting $\varepsilon \rightarrow 0^{+}$in (2.12), we derive that $\lim _{t \rightarrow \infty} u(t, r)=0$ when $h_{\infty}=R^{*}$. Combining this, (2.12) and (2.13), we have

$$
\lim _{t \rightarrow \infty} u(t, r)=0 \quad \text { when } h_{\infty} \leq R^{*}
$$

or, when $h_{\infty}>R^{*}$,

$$
\lim _{t \rightarrow \infty} u(t, r)=u^{*}(r) \quad \text { locally uniformly in }\left(R, h_{\infty}\right),
$$

where $u^{*}(r)$ is the unique positive solution of

$$
\left\{\begin{array}{l}
\Delta v+v(1-v)=0, \quad R<r<h_{\infty} \\
v\left(h_{\infty}\right)=v(R)=0 .
\end{array}\right.
$$

We now show that (2.15) is impossible when $h_{\infty}>R$. Suppose on the contrary that (2.15) holds when $h_{\infty}>R$. Since $h_{\infty} \leq R^{*}$, then there exists $H>0$ such that $h(t) \leq H$ for $t>0$. Using the approach of proving $T<+\infty$ in (ii), we can show that $\lim _{t \rightarrow T} h(t)=R$ for some $0<T<+\infty$, this contradicts 
the assumption $h_{\infty}>R$. Hence $\lim _{t \rightarrow \infty} u(t, r)=u^{*}(r)$ locally uniformly in $\left(R, h_{\infty}\right)$.

We next prove that $h_{\infty} \equiv l_{\alpha}$ and $u^{*}(r) \equiv v_{\alpha}(r)$ for $r \in\left[R, l_{\alpha}\right]$. Applying $L^{p}$ estimates as well as the Sobolev embedding theorem we conclude that,

$$
\left\|u(t, \cdot)-u^{*}(\cdot)\right\|_{C^{1+\frac{\gamma}{2}}([R, h(t)])} \rightarrow 0(t \rightarrow \infty)
$$

for some $\gamma \in(0,1)$. It follows that $h(t) h^{\prime}(t)=-u_{r}(t, h(t))-\alpha \rightarrow-\left(u^{*}\right)^{\prime}\left(h_{\infty}\right)-$ $\alpha$, as $t \rightarrow \infty$. On the other hand, $h(t)$ is Hölder continuous and $h_{\infty}<+\infty$, so $h^{\prime}(t) \rightarrow 0$. Hence $-\left(u^{*}\right)^{\prime}\left(h_{\infty}\right)=\alpha$. By the uniqueness of the solution of (1.7) and (2.16), we have $h_{\infty} \equiv l_{\alpha}$ and $u^{*}(r) \equiv v_{\alpha}(r)$ or $R \leq r \leq h_{\infty}$.

Remark 5. The approaches used in the the proof of Theorem 1 (i) remain true when $\alpha \leq 0$, and the arguments used in the the proof of Theorem 1 (ii)-(iii) are also valid for $\alpha \geq \alpha_{0}$.

Remark 6. Assume $T<+\infty$, then $\lim _{t \rightarrow T} h(t)=R$, which implies vanishing. Indeed, assume on the contrary that $\inf _{0<t<T}[h(t)-R]>0$, then by standard $L^{p}$ estimates, the Sobolev embedding theorem and the Hölder estimates for parabolic equations, we can extend the solution to some interval $(0, \bar{T})$ with $\bar{T}>T$ as long as $\inf _{0<t<T}[h(t)-R]>0$, this is a contradiction since $(0, T)$ is the maximal existence interval of the solution $u(t, x)$. Therefore, when spreading and transition happens, we have $T=+\infty$.

\subsection{Proof of Theorem 2.}

(i) Assume $\alpha \geq \alpha_{0}$, we prove that only vanishing happens for the solution $(u, h)$ of (1.1) defined on the maximal time interval $[0, T)$ with $T \in(0,+\infty]$. Let $q$ be the unique positive solution of

$$
\left\{\begin{array}{l}
q_{r r}+q(1-q)=0, \quad r>R \\
q(R)=0, q(+\infty)=1, q(r)>0, r>R
\end{array}\right.
$$

One can show that $q^{\prime}(R)=\alpha_{0}$. For some $M, \sigma>0, \delta \in(0,1), \hat{R}>R+\sigma M$ to be determined below, we define

$\bar{h}(t):=\hat{R}-\sigma M e^{-\delta t}, \quad \bar{u}(t, r):=\left(1+M e^{-\delta t}\right) q(\bar{h}(t)-r+R), R<r<\bar{h}(t), t>0$.

Obviously, for any $t>0$,

$$
\bar{u}(t, \bar{h}(t))=0 \quad \text { and } \quad \bar{u}(t, R)>0=u(t, R) .
$$

Based on the property that $q^{\prime}(r)>0$ for $r>R$, one can calculate directly as in [10] to show that, when $\sigma>0$ is large,

$$
\bar{u}_{t}-\bar{u}_{r r}-\frac{N-1}{r} \bar{u}_{r}-\bar{u}(1-\bar{u}) \geq \bar{u}_{t}-\bar{u}_{r r}-\bar{u}(1-\bar{u}) \geq 0 .
$$

On the other hand, when $\sigma>0$ is large we have

$$
\bar{h}(t) \bar{h}^{\prime}(t)=\left(\hat{R}-\sigma M e^{-\delta t}\right) M \sigma \delta e^{-\delta t} \geq R M \sigma \delta e^{-\delta t} \geq-\bar{u}_{r}(t, \bar{h}(t))-\alpha .
$$


In the above last inequality, we have used the facts $q^{\prime}(R)=\alpha_{0}, \alpha \geq \alpha_{0}$ and $-\bar{u}_{r}(t, \bar{h}(t))-\alpha=\left(1+M e^{-\delta t}\right) q^{\prime}(R)-\alpha=M e^{-\delta t} \alpha_{0}+\alpha_{0}-\alpha \leq M e^{-\delta t} \alpha_{0}$.

Moreover, we can choose large $\hat{R}$ and $M>0$ such that $\bar{h}(0)>h(0)$ and

$$
\bar{u}(0, r)=(1+M) q(\bar{h}(0)-r+R) \geq u_{0}(r), r \in\left[R, h_{0}\right] .
$$

Hence, by (2.17)-(2.20), one can show that $(\bar{u}, \bar{h})$ is an upper solution of the problem (1.1) in $Q:=\{(t, r): R<r<h(t), t>0\}$. Therefore, it follows from the comparison principle Lemma 1 that $u(t, r) \leq \bar{u}(t, r)$ in $Q$ and $h(t) \leq \bar{h}(t)$ for $t \in[0, T)$. From this we have $h_{\infty}<+\infty$. We next show that $h_{\infty}=R$, suppose on the contrary that $R<h_{\infty}<+\infty$, then using the similar arguments as in the proof of Theorem 1(iii), we can derive that $u$ converges to $v_{\alpha}$, this is impossible since (1.7) has no positive solution when $\alpha \geq \alpha_{0}$ (see Section 3 in [13] for details). Hence $h_{\infty}=R$. It then follows from the proof of Theorem 1 (ii) that vanishing happens.

(ii) Assume $\alpha<0$, then only spreading happens for the solution $(u, h)$ of (1.1). By the Hopf lemma, we have $u_{r}(t, h(t))<0$, hence $h(t) h^{\prime}(t)=$ $-u_{r}(t, h(t))-\alpha>-\alpha>0$. Therefore, $h(t) \rightarrow+\infty$ as $t \rightarrow \infty$. It then follows from Theorem 1 (i) that spreading happens.

(iii) We only consider the case $h_{\infty}<+\infty$ since the spreading phenomenon $\left(h_{\infty}=+\infty\right)$ follows from Theorem 1 (i). The proof is similar as that in [5, Lemma 2.2] and Theorem (iii), for reader's convenience, we give the proof and divide it into two steps.

Step 1. We show that if $h_{\infty}<+\infty$, then $h_{\infty} \leq R^{*}$. Assume on the contrary that $h_{\infty}>R^{*}$, then there exists $T_{1}>0$ such that $h(t)>R^{*}$ for all $t>T_{1}$. Thus the first eigenvalue $\lambda_{1}$ of (1.8) with $l=h(t)$ satisfies $\lambda_{1}<1$ for all $t>T_{1}$. Moreover, by the definition of $h_{\infty}$, for any small $\varepsilon>0$, there is $T_{2}=T_{2}(\varepsilon)>T_{1}$ such that,

$$
R^{*}<h_{\infty}-\varepsilon<h(t)<h_{\infty}+\varepsilon, \quad t \geq T_{2} .
$$

Consider the problem

$$
\begin{cases}\underline{u}_{t}=\Delta \underline{u}+\underline{u}(1-\underline{u}), & t>T_{2}, R<r<h_{\infty}-\varepsilon, \\ \underline{u}(t, R)=\underline{u}\left(t, h_{\infty}-\varepsilon\right)=0, & t>T_{2}, \\ \underline{u}\left(T_{2}, r\right)=u\left(T_{2}, r\right), & R<r<h_{\infty}-\varepsilon .\end{cases}
$$

Since $\lambda_{1}<1$, the problem (2.21) has a unique positive solution $\underline{u}_{\varepsilon}(t, r)$ (see, Corollary 3.4 in [3]), and

$$
\underline{u}_{\varepsilon}(t, r) \rightarrow \underline{u}_{\varepsilon}^{*}(r) \text { in } C^{2}\left(\left[R, h_{\infty}-\varepsilon\right]\right) \text { as } t \rightarrow \infty,
$$

where $\underline{u}_{\varepsilon}^{*}(r)$ is the unique positive radially symmetric solution of the problem (2.14). Meanwhile, by the comparison principle we have $u(t, r) \geq \underline{u}_{\varepsilon}(t, r)$ for $t>T_{2}$ and $r \in\left[R, h_{\infty}-\varepsilon\right]$. Therefore,

$$
\liminf _{t \rightarrow \infty} u(t, r) \geq \underline{u}_{\varepsilon}^{*}(r) \quad \text { for } r \in\left[R, h_{\infty}-\varepsilon\right] .
$$

On the other hand, consider the problem

$$
\begin{cases}w_{t}=\Delta w+w(1-w), & t>T_{2}, R<r<h_{\infty}+\varepsilon, \\ w(t, R)=w\left(t, h_{\infty}+\varepsilon\right)=0, & t>T_{2}, \\ w\left(T_{2}, r\right) \geq \widetilde{u}\left(T_{2}, r\right), & R<r<h_{\infty}+\varepsilon,\end{cases}
$$


where

$$
\widetilde{u}\left(T_{2}, r\right)= \begin{cases}u\left(T_{2}, r\right) & R<r<h\left(T_{2}\right), \\ 0, & h\left(T_{2}\right) \leq r \leq h_{\infty}+\varepsilon .\end{cases}
$$

Similarly, the problem (2.23) admits a unique positive solution $w_{h_{\infty}+\varepsilon}(t, r)$ and it converges to $\bar{u}_{\varepsilon}^{*}(r)$ as $t \rightarrow \infty$, where $\bar{u}_{\varepsilon}^{*}(r)$ is the unique positive solution of the problem (2.11). Meanwhile, by the comparison principle, we have $u(t, r) \leq$ $w_{h_{\infty}+\varepsilon}(t, r)$ for $t>T_{2}$ and $r \in[R, h(t)]$. Hence,

$$
\limsup _{t \rightarrow \infty} u(t, r) \leq \bar{u}_{\varepsilon}^{*}(r) \text { for } r \in\left[R, h_{\infty}\right] .
$$

By (2.22), (2.24), a standard compactness and uniqueness argument, we have

$$
\lim _{t \rightarrow \infty} u(t, r)=u^{*}(r) \quad \text { for } r \in\left[R, h_{\infty}\right),
$$

where $u^{*}(r)$ is the solution of (2.16). Moreover,

$$
h(t) h^{\prime}(t)=-u_{r}(t, h(t)) \rightarrow-\left(u^{*}\right)^{\prime}(r)\left(h_{\infty}\right)>0 \quad \text { as } t \rightarrow \infty,
$$

this implies that $h_{\infty}=\infty$, which contradicts our assumption that $h_{\infty}<\infty$. Therefore we must have $h_{\infty} \leq R^{*}$.

Step 2. We now show that $\lim _{t \rightarrow \infty} \max _{R<r<h(t)} u(t, r)=0$. Consider (2.23) again, since $h_{\infty} \leq R^{*}$ and $\varepsilon$ is arbitrary small, then $\lim _{t \rightarrow \infty} w_{h_{\infty}+\varepsilon}(t, r)=\bar{u}_{\varepsilon}^{*}(r) \rightarrow 0$ as $\varepsilon \rightarrow 0^{+}$. Therefore, we have

$$
\lim _{t \rightarrow \infty} \max _{R<r<h(t)} u(t, r)=0 .
$$

\subsection{Proof of Theorem 3.}

In order to give the proof, we need to consider the following problem in one dimensional space:

$$
\left\{\begin{array}{l}
q^{\prime \prime}+\gamma q^{\prime}+q(1-q)=0, \quad R<x<+\infty \\
q(R)=0, \quad q(+\infty)=1 .
\end{array}\right.
$$

By [13], for any $\gamma \in(-2,2)$, the problem $(2.25)$ has a unique solution $\left(\gamma, q_{\gamma}(x)\right)$ with the property that $q_{\gamma}^{\prime}(x)>0$ for $x \geq R$. Especially, when $\gamma=0$, denote the solution by $q_{0}(x)$. By the definition of $\alpha_{0}$, we see that $\alpha_{0}=q_{0}^{\prime}(R)$. Moreover, for any small $\varepsilon>0$, there exists $\gamma_{\varepsilon}<0$ such that the problem (2.25) has a unique positive solution $q_{\gamma_{\varepsilon}}(x)$ with $q_{\gamma_{\varepsilon}}^{\prime}(R)=\alpha_{0}-\varepsilon$.

We first construct an upper solution. For $t>1, \delta \in(0,1)$ and some $M, R_{1}, \sigma>0$ to be determined later, we define

$$
\begin{aligned}
& \bar{h}(t):=\sqrt{2\left(\alpha_{0}-\alpha\right) t}+h(1)-M \sigma e^{-\delta t}+R_{1}, \quad t \geq 1 \\
& \bar{u}(t, r)=\left(1+M e^{-\delta t}\right) q_{0}(\bar{h}(t)-r+R), R<r<\bar{h}(t), \quad t \geq 1 .
\end{aligned}
$$

We now check that $(\bar{u}, \bar{h})$ is an upper solution of (1.1) for large $M, R_{1}, \sigma$ with $R_{1}>M \sigma e^{-\delta}$. Clearly, for any $t \geq 1$,

$$
\bar{u}(t, R)=\left(1+M e^{-\delta t}\right) q_{0}(\bar{h}(t))>0=u(t, R), \quad \bar{u}(t, \bar{h}(t))=0 .
$$


Choose $M$ and $R_{1}$ sufficiently large with $R_{1}>M \sigma e^{-\delta}$ such that

$$
\bar{u}(1, r)=\left(1+M e^{-\delta}\right) q_{0}(\bar{h}(1)-r+R)>u(1, r) \quad \text { for } R<r<h(1) .
$$

By the definition of $\bar{h}(t), R_{1}>M \sigma e^{-\delta}$, we have that

$$
\begin{aligned}
& \bar{h}(t) \bar{h}^{\prime}(t)=\left(\sqrt{2\left(\alpha_{0}-\alpha\right) t}+h(1)-M \sigma e^{-\delta t}+R_{1}\right) \\
& \quad \times\left(\frac{1}{2} \sqrt{2\left(\alpha_{0}-\alpha\right)} t^{-\frac{1}{2}}+M \sigma \delta e^{-\delta t}\right) \geq \alpha_{0}-\alpha+M \sigma \delta e^{-\delta t} \sqrt{2\left(\alpha_{0}-\alpha\right) t} .
\end{aligned}
$$

Moreover,

$$
\begin{aligned}
-\bar{u}_{r}(t, \bar{h}(t))-\alpha & =\left(1+M e^{-\delta t}\right) q_{0}^{\prime}(R)-\alpha \\
& =\left(1+M e^{-\delta t}\right) \alpha_{0}-\alpha=\alpha_{0}-\alpha+M e^{-\delta t} \alpha_{0} .
\end{aligned}
$$

Hence if we take $\sigma>0$ such that $\sigma \delta \sqrt{2\left(\alpha_{0}-\alpha\right)} \geq \alpha_{0}$, then

$$
\bar{h}(t) \bar{h}^{\prime}(t) \geq-\bar{u}_{r}(t, \bar{h}(t))-\alpha .
$$

Using the similar arguments as in [10, Lemma 3.2] and the property $q_{0}^{\prime}(r)>$ 0 for $r \in[R,+\infty)$, we can show that, for $t \geq 1$,

$$
\bar{u}_{t}-\frac{N-1}{r} \bar{u}_{r}-\bar{u}_{r r}-\bar{u}(1-\bar{u}) \geq \bar{u}_{t}-\bar{u}_{r r}-\bar{u}(1-\bar{u}) \geq 0 .
$$

By the definition of the upper solution and (2.26)-(2.29), we see that $(\bar{u}, \bar{h})$ is an upper solution of (1.1) for $t \geq 1$. Hence

$$
h(t) \leq \bar{h}(t) \text { for all } t \geq 1 .
$$

We now construct a lower solution. By the property of $V$ (the solution of (1.4)), for any sufficiently small $\varepsilon>0$, there exists $X>0$ such that $V(X)>$ $1-\varepsilon$. Choose $X_{1}>X$ such that $\frac{N-1}{X_{1}} \leq-\frac{\gamma_{\varepsilon}}{2}$, where $\left(\gamma_{\varepsilon}, q_{\gamma_{\varepsilon}}\right)$ is the unique solution of (2.25) with $q_{\gamma_{\varepsilon}}^{\prime}(R)=\alpha_{0}+\varepsilon$. By the monotonicity of $V$, there is $\nu>0$ sufficiently small such that

$$
V(X)<(1-\nu) V\left(X_{1}\right) .
$$

Define $c:=(1-2 \varepsilon)\left(\alpha_{0}-\varepsilon\right)-\alpha$. Since $\varepsilon>0$ is sufficiently small and $\alpha<\alpha_{0}$, one can show that $c>0$. Choose $T \gg 1$ sufficiently large such that

$$
\frac{\sqrt{2 c}}{2} T^{-\frac{1}{2}}<\frac{-\gamma_{\varepsilon}}{2}, e^{-\delta T} \leq \frac{\varepsilon}{2}, \sigma \sqrt{2 / c} e^{-\delta t}<t^{-\frac{1}{2}} \text { for } t \geq T,
$$

where $\delta \in\left(\frac{1}{2}, 1\right)$, and for all $t \geq T$,

$$
u(t, r) \geq(1-\nu) V\left(X_{1}\right) \text { for } r \in\left[X_{1}, 2 X_{1}\right] .
$$

The above inequalities follow from (1.3) in the spreading case and the monotonicity of $V$. For some large $\sigma>0$ and a small $M_{1}\left(0<M_{1} \ll 1\right)$ satisfying $M_{1} \sigma \sqrt{2 / c}<T^{-\frac{1}{2}}$ to be determined below, we define

$$
\underline{h}(t):=\sqrt{2 c t}+M_{1} \sigma e^{-\delta t}-H_{0}, t \geq T,
$$




$$
\underline{u}(t, r):=\left[V(X)-\varepsilon-M_{1} e^{-\delta t}\right] q_{\gamma_{\varepsilon}}(\underline{h}(t)-r+R), X_{1}<r<\underline{h}(t), t \geq T,
$$

where $H_{0}$ is a constant satisfying

$$
\sqrt{2 c T}+M_{1} \sigma e^{-\delta T}-H_{0} \in\left(X_{1}, 2 X_{1}\right]
$$

We next check that $(\underline{u}, \underline{h})$ is a lower solution. Clearly,

$$
\underline{u}(t, \underline{h}(t))=0 \quad \text { for } t \geq T .
$$

By (2.31) and (2.33) we have

$\underline{u}\left(t, X_{1}\right) \leq V(X)-\varepsilon-M_{1} e^{-\delta t} \leq V(X)<(1-\nu) V\left(X_{1}\right) \leq u\left(t, X_{1}\right) \quad$ for $t \geq T$.

Direct computations yield that

$$
\begin{aligned}
& -\underline{u}_{r}(t, \underline{h}(t))-\alpha=\left[V(X)-\varepsilon-M_{1} e^{-\delta t}\right] q_{\gamma_{\varepsilon}}^{\prime}(R)-\alpha \\
& \quad \geq\left[1-2 \varepsilon-M_{1} e^{-\delta t}\right] q_{\gamma_{\varepsilon}}^{\prime}(R)-\alpha \geq c-M_{1} e^{-\delta t} q_{\gamma_{\varepsilon}}^{\prime}(R) .
\end{aligned}
$$

Here, we have used the fact that $V(X)>1-\varepsilon$ and $q_{\gamma_{\varepsilon}}^{\prime}(R)=\alpha_{0}-\varepsilon$. Moreover, note that $M_{1} \sigma \sqrt{2 / c}<T^{-\frac{1}{2}}, \delta \in\left(\frac{1}{2}, 1\right)$, then by the definition of $\underline{h}$, we have

$$
\underline{h}(t) \underline{h}^{\prime}(t) \leq c+M_{1} \sigma e^{-\delta t}\left(-\sqrt{2 c t} \delta+\sqrt{c / 2} t^{-\frac{1}{2}}\right) \leq c+M_{1} \sigma e^{-\delta t}(-\sqrt{2 c} \delta+\sqrt{c / 2}) .
$$

Hence, if we take $\sigma>0$ such that $\sigma(\sqrt{2 c} \delta-\sqrt{c / 2})>q_{\gamma_{\varepsilon}}^{\prime}(R)$, then

$$
\underline{h}(t) \underline{h}^{\prime}(t) \leq-\underline{u}_{r}(t, \underline{h}(t))-\alpha \text { for } t \geq T \text {. }
$$

By $(2.31),(2.33),(2.34)$ and the definition of $\underline{u}$, one can derive that $\underline{u}(T, r) \leq$ $V(X) \leq(1-\nu) V\left(X_{1}\right) \leq u(T, r)$ for $r \in\left[X_{1}, \underline{h}(T)\right]$.

Finally, using the similar arguments as in [10, Lemma 3.3] and (2.32), one can calculate directly

$$
\begin{aligned}
\underline{u}_{t} & -\frac{N-1}{r} \underline{u}_{r}-\underline{u}_{r r}-\underline{u}(1-\underline{u})=M_{1} \delta e^{-\delta t} q_{\gamma_{\varepsilon}}+\left(\frac{\sqrt{2 c}}{2} t^{-\frac{1}{2}}+\frac{N-1}{r}\right) \\
& \times\left[V(X)-\varepsilon-M_{1} e^{-\delta t}\right] q_{\gamma_{\varepsilon}}^{\prime}-M_{1} \sigma \delta e^{-\delta t}\left[V(X)-\varepsilon-M_{1} e^{-\delta t}\right] q_{\gamma_{\varepsilon}}^{\prime}-[V(X)-\varepsilon \\
& \left.-M_{1} e^{-\delta t}\right] q_{\gamma_{\varepsilon}}^{\prime \prime}-\left[V(X)-\varepsilon-M_{1} e^{-\delta t}\right] q_{\gamma_{\varepsilon}}\left(1-\left[V(X)-\varepsilon-M_{1} e^{-\delta t}\right] q_{\gamma_{\varepsilon}}\right) \\
\leq & M_{1} \delta e^{-\delta t} q_{\gamma_{\varepsilon}}-\gamma_{\varepsilon}\left[V(X)-\varepsilon-M_{1} e^{-\delta t}\right] q_{\gamma_{\varepsilon}}^{\prime}-M_{1} \sigma \delta e^{-\delta t}\left[V(X)-\varepsilon-M_{1} e^{-\delta t}\right] q_{\gamma_{\varepsilon}}^{\prime} \\
& -\left[V(X)-\varepsilon-M_{1} e^{-\delta t}\right] q_{\gamma_{\varepsilon}}^{\prime \prime}-\left[V(X)-\varepsilon-M_{1} e^{-\delta t}\right] q_{\gamma_{\varepsilon}}\{1-[V(X)-\varepsilon \\
& \left.\left.-M_{1} e^{-\delta t}\right] q_{\gamma_{\varepsilon}}\right\}=M_{1} \delta e^{-\delta t} q_{\gamma_{\varepsilon}}+\left[V(X)-\varepsilon-M_{1} e^{-\delta t}\right] q_{\gamma_{\varepsilon}}\left(1-q_{\gamma_{\varepsilon}}\right) \\
& -M_{1} \sigma \delta e^{-\delta t}\left[V(X)-\varepsilon-M_{1} e^{-\delta t}\right] q_{\gamma_{\varepsilon}}^{\prime} \\
& -\left[V(X)-\varepsilon-M_{1} e^{-\delta t}\right] q_{\gamma_{\varepsilon}}\left\{1-\left[V(X)-\varepsilon-M_{1} e^{-\delta t}\right] q_{\gamma_{\varepsilon}}\right\} \leq 0 .
\end{aligned}
$$

We used (2.32) in the above first inequality, and in the second equality we have used that $\left(\gamma_{\varepsilon}, q_{\gamma_{\varepsilon}}\right)$ is the solution of (2.25). Applying the method in [10, Lemma 3.3] and (2.32), one can show that the last inequality holds. 
By (2.35)-(2.38) and the definition of the lower solution (see Remark 4), we see that $(\underline{u}, \underline{h})$ is a lower solution of the problem (1.1). Hence

$$
h(t) \geq \underline{h}(t) \text { for } t>T .
$$

It follows from $(2.30),(2.39)$ and the definition of $c$ that

$$
\sqrt{2\left[(1-2 \varepsilon)\left(\alpha_{0}-\varepsilon\right)-\alpha\right]} \leq \liminf _{t \rightarrow \infty} \frac{h(t)}{\sqrt{t}} \leq \limsup _{t \rightarrow \infty} \frac{h(t)}{\sqrt{t}} \leq \sqrt{2\left(\alpha_{0}-\alpha\right)} .
$$

Since $\varepsilon>0$ can be arbitrarily small, we have

$$
\lim _{t \rightarrow \infty} h(t) / \sqrt{t}=\sqrt{2\left(\alpha_{0}-\alpha\right)} .
$$

\section{Sufficient Conditions}

In this section, we give some necessary and sufficient conditions for vanishing and spreading, and a sufficient condition for transition.

Proposition 1. Assume $0<\alpha<\alpha_{0}, h_{0}>0$ and $u_{0}(r) \in \mathcal{J}\left(h_{0}\right)$. Let $(u, h)$ be the solution of (1.1) with initial data $u_{0}(r)$. Then the following properties hold:

(i) vanishing happens if and only if there exists $t_{1}$ such that

$$
u\left(t_{1}, r\right)<v_{\alpha}(r) \text { for } r \in\left[R, h\left(t_{1}\right)\right]
$$

and $\left\|u\left(t_{1}, r\right)\right\|_{L^{\infty}\left(\left[R, h\left(t_{1}\right)\right]\right)}$ is sufficiently small;

(ii) spreading happens if and only if there exists $t_{2}$ such that

$$
u\left(t_{2}, r\right)>v_{\beta}(r) \quad \text { for } \quad r \in\left[R, l_{\beta}\right] \subset\left[R, h\left(t_{2}\right)\right],
$$

where $\beta \in\left(\alpha, \alpha_{0}\right),\left(l_{\beta}, v_{\beta}\right)$ is the unique positive solution of

$$
\left\{\begin{array}{l}
\Delta v+v(1-v)=0, \quad l_{\alpha}<r<L \\
v(R)=0, v(L)=0,-v^{\prime}(L)=\beta
\end{array}\right.
$$

(iii) transition happens if $h_{0}=l_{\alpha}$ and $u_{0}(r) \equiv v_{\alpha}(r)$ for $r \in\left[R, h_{0}\right]$.

Proof. (i) The condition (3.1) follows from the definition of vanishing. We only need to show that (3.1) is a sufficient condition for vanishing. By the comparison principle, we have

$$
u(t, r) \leq v_{\alpha}(r), r \in[R, h(t)], t>t_{1} .
$$

Hence $h(t) \leq l_{\alpha}$ for all $t>t_{1}$. Consider the problem (2.9) with $L:=2\left(l_{\alpha}+1\right)$, then it follows from the last part of the proof of Theorem 1 (ii) that $h(t)$ converges to $R$ within a finite time if $\left\|u\left(t_{1}, r\right)\right\|_{L^{\infty}\left(\left[R, h\left(t_{1}\right)\right]\right)}$ is sufficiently small. Combining this and Theorem 1 (ii), we conclude that vanishing happens. 
(ii) Clearly, inequality (3.2) is a necessary condition. We now prove that it is a sufficient condition for spreading. By the comparison principle in $[9$, Lemma 2.1] and $\alpha<\beta<\alpha_{0}$, one can show that (3.3) has a unique positive solution $\left(l_{\beta}, v_{\beta}\right)$ and $l_{\beta}>l_{\alpha}$. Then it follows from $\beta>\alpha$ and the definition of lower solution (see Remark 4) that $v_{\beta}(r)$ is an lower solution of (1.1) when $t>t_{2}$. Therefore, by the comparison principle Lemma 1 we have

$$
h(t)>l_{\beta}>l_{\alpha}, u(t, r)>v_{\beta}(r) \text { for } r \in\left[R, l_{\beta}\right], t>t_{2} .
$$

Hence

$$
h_{\infty}:=\lim _{t \rightarrow \infty} h(t) \geq l_{\beta}>l_{\alpha}
$$

By Theorem 1 (there are only three cases for $h_{\infty}: h_{\infty}=+\infty, h_{\infty}=R, h_{\infty}=$ $l_{\alpha}$ ) we have $h_{\infty}=+\infty$ and spreading happens.

(iii) By $u_{0}(r) \equiv v_{\alpha}(r)$ for $r \in\left[R, h_{0}\right]=\left[R, l_{\alpha}\right]$, one can verify directly that $h(t) \equiv l_{\alpha}$ and $u(t, r) \equiv v_{\alpha}(r)$ for all $t>0$ and $r \in\left[R, l_{\alpha}\right]$. Then it follows from Theorem 1(iii) that transition happens.

\section{Acknowledgement}

The research is sponsored by National Science Foundation of China (No. 11526134, 1150214 and 61203006), Natural Science Foundation of Shanghai (No. 13ZR1454900), and the Innovation Program of Shanghai Municipal Eduction Commission (No. 14ZZ151). The authors would like to thank the reviewers who give us helpful comments.

\section{References}

[1] J. Cai. Asymptotic behavior of solutions of Fisher-KPP equation with free boundary conditions. Nonlinear Analysis: Real World Applications, 16:170-177, 2014. https://doi.org/10.1016/j.nonrwa.2013.09.016.

[2] J. Cai, B. Lou and M. Zhou. Asymptotic behavior of solutions of a reaction diffusion equation with free boundary conditions. Journal of Dynamics and Differential Equations, 26(4):1007-1028, 2014. https://doi.org/10.1007/s10884014-9404-z.

[3] R.S. Cantrell and Ch. Cosner. Spatial Ecology via Reaction-diffusion Equations. John Wiley \& Sons Ltd., Chichester, UK, 2003.

[4] S. Cui and A. Friedman. Analysis of a mathematical model of protocell. Journal of Mathematical Analysis and Applications, 236(1):171-206, 1999. https://doi.org/10.1006/jmaa.1999.6444.

[5] Y. Du and Z. Guo. Spreading-vanishing dichotomy in a diffusive logistic model with a free boundary, II. Journal of Differential Equations, 250(12):4336-4366, 2011. https://doi.org/10.1016/j.jde.2011.02.011.

[6] Y. Du and Z. Guo. The Stefan problem for the Fisher-KPP equation. Journal of Differential Equations, 253(3):996-1035, 2012. https://doi.org/10.1016/j.jde.2012.04.014.

[7] Y. Du and Z. Lin. Spreading-vanishing dichotomy in the diffusive logistic model with a free boundary. SIAM Journal on Mathematical Analysis, 42(1):377-405, 2010. https://doi.org/10.1137/090771089. 
[8] Y. Du and B. Lou. Spreading and vanishing in nonlinear diffusion problems with free boundaries. Journal of the European Mathematical Society, 17(10):26732724, 2015. https://doi.org/10.4171/JEMS/568.

[9] Y. Du and L. Ma. Logistic type equations on $\mathbb{R}^{n}$ by a squeezing method involving boundary blow-up solutions. Journal of the London Mathematical Society, 64(1):107-124, 2001. https://doi.org/10.1017/S0024610701002289.

[10] Y. Du, H. Matsuzawa and M. Zhou. Sharp estimate of the spreading speed determined by nonlinear free boundary problems. SIAM Journal on Mathematical Analysis, 46(1):375-396, 2014. https://doi.org/10.1137/130908063.

[11] A. Friedman. Partial Differential Equations of Parabolic Type. Dover Publications, USA, 2008.

[12] A. Friedman and B. Hu. A Stefan problem for a protocell model. SIAM Journal on Mathematical Analysis, 30(4):912-926, 1999. https://doi.org/10.1137/S0036141098337588.

[13] H. Gu, B. Lou and M. Zhou. Long time behavior of solutions of Fisher-KPP equation with advection and free boundaries. Journal of Functional Analysis, 269(6):1714-1768, 2015. https://doi.org/10.1016/j.jfa.2015.07.002.

[14] D. Hilhorst, M. Iida, M. Mimura and H. Ninomiya. A competitiondiffusion system approximation to the classical two-phase Stefan problems. Japan Journal of Industrial and Applied Mathematics, 18:161-180, 2001. https://doi.org/10.1007/BF03168569.

[15] D. Hilhorst, M. Iida, M. Mimura and H. Ninomiya. Relative compactness in $L^{p}$ of solutions of some $2 \mathrm{~m}$ components competition-diffusion systems. Discrete and Continuous Dynamical Systems - Series A, 21(1):233-244, 2008. https://doi.org/10.3934/dcds.2008.21.233.

[16] Y. Kaneko. Spreading and vanishing behaviors for radially symmetric solution of free boundary problems for reaction-diffusion equations. Nonlinear Analysis: Real World Applications, 18:121-140, 2014. https://doi.org/10.1016/j.nonrwa.2014.01.008.

[17] Y. Kaneko and Y. Yamada. A free boundary problem for a reaction-diffusion equation appearing in ecology. Advances in Mathematical Sciences and Applications, 21(2):467-492, 2011.

[18] H. Schwegler, K. Tarumi and B. Gerstmann. Physico-chemical model of a protocell. Journal of Mathematical Biology, 22(3):335-348, 1985. https://doi.org/10.1007/BF00276490. 